\title{
Total weight choosability of graphs
}

\author{
Jakub Przybyło \\ AGH University of Science and Technology \\ Al. Mickiewicza 30, 30-059 Kraków, Poland \\ przybylo@wms.mat.agh.edu.pl \\ Mariusz Woźniak \\ AGH University of Science and Technology \\ Al. Mickiewicza 30, 30-059 Kraków, Poland \\ mwozniak@agh. edu.pl
}

Submitted: Jan 26, 2009; Accepted: May 5, 2011; Published: May 16, 2011

Mathematics Subject Classifications: 05C78

\begin{abstract}
Suppose the edges and the vertices of a simple graph $G$ are assigned $k$-element lists of real weights. By choosing a representative of each list, we specify a vertex colouring, where for each vertex its colour is defined as the sum of the weights of its incident edges and the weight of the vertex itself. How long lists ensures a choice implying a proper vertex colouring for any graph? Is there any finite bound or maybe already lists of length two are sufficient? We prove that 2-element lists are enough for trees, wheels, unicyclic and complete graphs, while the ones of length 3 are sufficient for complete bipartite graphs. Our main tool is an algebraic theorem by Alon called Combinatorial Nullstellensatz.
\end{abstract}

Keywords: graph labelling, neighbour distinguishing total weighting, total list weighting, vertex colouring

\section{Introduction}

Let $G=(V, E)$ be a simple graph. Given its total weighting $w: V \cup E \rightarrow \mathbb{R}$, we define a total weight for each vertex $v \in V$ as $t_{w}(v):=w(v)+\sum_{e \ni v} w(e)$. We say that $G$ is $\{1, \ldots, k\}$-totaly weight colourable if there is a total weighting $w: V \cup E \rightarrow\{1, \ldots, k\}$ that is neighbour distinguishing (or vertex colouring), i.e. such that $t_{w}(u) \neq t_{w}(v)$ for every edge $u v$ of $G$. The problem of finding the smallest $k$ for which $G$ is $\{1, \ldots, k\}$-totaly weight colourable was first studied in [14] and [11] as a variant of a similar problem for 
edge weightings introduced in [9] by Karoński, Łuczak and Thomason (where we do not weight vertices, hence do not add their weights to the sums of their incident edges, see also [1]). Przybyło and Woźniak even conjectured that $k=2$ is enough for all graphs, but only managed to prove that the weights $1, \ldots, 11$ are always sufficient to construct a desired total weighting, see [14]. In the same paper, the stated conjecture was verified for several classes of graphs, including the complete, 3-colourable and 4-regular graphs. Recently Kalkowski [8] discovered an elegant algorithm which solves our problem using weights 1,2,3 for the edges and 1,2 for the vertices, but the conjecture remained unsolved.

An inspiration for our research are the similar problems, where in turn all the vertices (not only the neighbouring ones) are distinguished by total, [3, 13], or edge weightings, $[6,7,10,12,13]$. Especially the second of them is devoted to investigations over a well known graph invariant, called irregularity strength, which has already been studied in over 40 articles.

In this paper we want to present a new direction in our quest for an appropriate method to attack the mentioned conjecture. For this reason, similarly as the authors of [4] in the case of edge weightings, we will consider the following list version of the problem and an algebraic approach to solving it. Let each edge $e \in E$ and each vertex $v \in V$ of a graph $G$ be assigned a list of $k$ real weights, $L_{e}, L_{v}$, and let $L$ be a collection of all these lists. We say that $G$ is totally weight choosable from these lists (or from $L$ ) if there is a neighbour distinguishing total weighting $w: V \cup E \rightarrow \bigcup_{L_{a} \in L} L_{a}$ such that $w(e) \in L_{e}$, $w(v) \in L_{v}$ for each $e \in E$ and each $v \in V$. A graph $G$ is $k$-totally weight choosable if it is totally weight choosable from any family of lists of size $k$.

Conjecture 1 Each graph is 2-totally weight choosable.

This conjecture is obviously stronger then the one stated in [14] by Przybyło and Woźniak (for non-list version of the problem), but its structure admits an application of Alon's algebraic method. The main aim of this paper is to transform the stated problem to an algebraic form suitable for using the theorem called Combinatorial Nullstellensatz, and to create a number of tools, helpful in our investigations, based on permanents of special matrices, see the next section. Having these prepared, we then prove that conjecture 1 holds for trees, cycles, unicyclic graphs and wheels, and that 3-element lists are enough for complete bipartite graphs. In the last section we present a proof of conjecture 1 for complete graphs, which does not use an algebraic method. It is also worth mentioning here that similar surveys has recently been in parallel conducted by Wong, Zhu et al., see $[5,15,16]$ for details.

\section{Algebraic model and tools}

The whole idea is based on the following theorem by Alon.

Theorem 2 (Combinatorial Nullstellensatz, [2]) Let $\mathbb{F}$ be an arbitrary field, and let $P=P\left(x_{1}, \ldots, x_{l}\right)$ be a polynomial in $\mathbb{F}\left[x_{1}, \ldots, x_{l}\right]$. Suppose the degree $\operatorname{deg}(P)$ of 
$P$ is $\sum_{i=1}^{l} k_{i}$, where each $k_{i}$ is a non-negative integer, and suppose the coefficient of $x_{1}^{k_{1}} \ldots x_{l}^{k_{l}}$ in $P$ is non-zero. Then if $S_{1}, \ldots, S_{l}$ are subsets of $\mathbb{F}$ with $\left|S_{i}\right|>k_{i}$, there are $s_{1} \in S_{1}, \ldots, s_{l} \in S_{l}$ so that $P\left(s_{1}, \ldots, s_{l}\right) \neq 0$.

Let $E(G)=\left\{e_{1}, \ldots, e_{m}\right\}$ be the set of edges and $V(G)=\left\{v_{1}, \ldots, v_{n}\right\}$ the set of vertices of a simple graph $G$. Associate each edge $e_{i}$ with a variable $x_{i}$, and each vertex $v_{j}$ with a variable $x_{m+j}$. Denote $X_{v_{j}}=\sum_{e_{i} \ni v_{j}} x_{i}$ and $Y_{v_{j}}=x_{m+j}+X_{v_{j}}$ for each $v_{j} \in V(G)$. Then choose any orientation of $G$ and define two polynomials $P_{G}$ and $T_{G}$ (first of which was defined in [4] to investigate an edge version of the problem) in variables $x_{1}, \ldots, x_{l}$, where $l=m+n$, by

$$
\begin{aligned}
& P_{G}\left(x_{1}, \ldots, x_{l}\right)=\prod_{(u, v) \in E(G)}\left(X_{v}-X_{u}\right), \\
& T_{G}\left(x_{1}, \ldots, x_{l}\right)=\prod_{(u, v) \in E(G)}\left(Y_{v}-Y_{u}\right) .
\end{aligned}
$$

Now given a polynomial $P \in \mathbb{F}\left[x_{1}, \ldots, x_{l}\right]$ and any monomial $M=c x_{1}^{k_{1}} \ldots x_{l}^{k_{l}}$ in its expansion, where $c \neq 0$, let $h(M)$ be the highest exponent of a variable in $M$. Define the monomial index of $P$ as the minimum of $h(M)$ taken over all non-vanishing monomials $M$ in $P$ and denote it as $\operatorname{mind}(P)$. Note that different orientations of a graph give the same, up to the sign, polynomials $P_{G}$ and $T_{G}$. Therefore, $\operatorname{mind}\left(P_{G}\right)$, denoted by $\operatorname{mind}(G)$, and $\operatorname{mind}\left(T_{G}\right)$, denoted by $\operatorname{tmind}(G)$, can be considered as graph invariants. We shall call them respectively the monomial index and the total monomial index of a graph. (Note that considering mind $(G)$ we must assume that $G$ does not contain an isolated edge, since otherwise $P_{G} \equiv 0$.) By theorem 2, the following holds.

Corollary 3 If $\operatorname{tmind}(G) \leq k$, then $G$ is $(k+1)$-totally weight choosable.

Note that it is not obvious (not known so far) that a given graph $G$ is $k$-totally weight choosable if the same holds for the edge (not total) version of the problem from [4] (though this fact is trivial for their non-list equivalents). The following however is not difficult to observe.

Observation 4 For each graph $G$ without an isolated edge, $\operatorname{tmind}(G) \leq \operatorname{mind}(G)$.

Proof. Assume that $G$ is of size $m$. Note that $T_{G}=P_{G}+R$, where $P_{G} \in \mathbb{F}\left[x_{1}, \ldots, x_{m}\right]$ and each monomial $M$ in the expansion of $R$ with non-zero coefficient must contain $x_{i}$ with $i>m$. Therefore $\operatorname{mind}\left(T_{G}\right)=\min \left\{\operatorname{mind}\left(P_{G}\right), \operatorname{mind}(R)\right\} \leq \operatorname{mind}\left(P_{G}\right)$.

In view of conjecture 1 , we would be pleased if we could show that $\operatorname{tmind}(G)=1$ for each graph $G$. In other words, we must prove that in the expansion of $T_{G}$ there is a non-vanishing monomial which is a product of $(m)$ different variables. It is however not even known if there is any absolute constant $k_{0}$ such that $\operatorname{tmind}(G) \leq k_{0}$ for each graph $G$. To estimate this graph invariant, we shall use a matrix associated with the polynomial 
$T_{G}$ and estimate its permanent index. Recall then first that for a given square matrix $A=\left(a_{i j}\right)$ of size $m$, the permanent of $A$ is defined as

$$
\operatorname{per} A=\sum_{\sigma} a_{1 \sigma(1)} a_{2 \sigma(2)} \ldots a_{m \sigma(m)}
$$

where $\sigma$ runs through all permutations of the set $\{1, \ldots, m\}$. On the other hand, the permanent rank of a not necessarily square matrix $A$ is the size of its largest square submatrix with non-zero permanent. Moreover, let $A^{(k)}=[A, \ldots, A]$ be a matrix formed of $k$ consecutive copies of the matrix $A$ of size $m \times l$. Now the permanent index of $A$ is the smallest $k$, for which the permanent rank of $A^{(k)}$ equals $m$. If it exists, we denote it by $\operatorname{pind}(A)$. For the remaining matrices we set $\operatorname{pind}(A)=\infty$. An alternative definition of this parameter goes as follows. Let $K=\left(k_{1}, \ldots, k_{l}\right)$ be a sequence of non-negative integers. Denote the columns of $A$ by $A_{1}, \ldots, A_{l}$, and denote by $A(K)$ a matrix obtained from $A$ by repeating $k_{i}$ times each subsequent column $A_{i}$. Then $\operatorname{pind}(A)$ is equal to the smallest $k$ for which exists a sequence $K=\left(k_{1}, \ldots, k_{l}\right)$ of non-negative integers such that $k_{1}+\ldots+k_{l}=m$, per $A(K) \neq 0$ and $k_{i} \leq k$ for $i=1, \ldots, l$.

Let $G=(V, E)$ be a graph with a given orientation, where $V=\left\{v_{1}, \ldots, v_{n}\right\}, E=$ $\left\{e_{1}, \ldots, e_{m}\right\}, l=n+m$. So that we could make use of the introduced notations, let us define a matrix $A_{G}=\left(a_{i j}\right)$ associated with $G$ as follows:

$$
a_{i j}=\left\{\begin{aligned}
1, & \text { if } e_{j} \text { is incident with the end of } e_{i} \text { or } v_{j-m} \text { is the end of } e_{i}, \\
-1, & \text { if } e_{j} \text { is incident with the begining of } e_{i} \text { or } v_{j-m} \text { is the begining } \\
& \text { of } e_{i}, \\
0, & \text { if } e_{j} \text { and } e_{i} \text { are not incident and } v_{j-m} \text { is neither the begining } \\
& \text { nor the end of } e_{i},
\end{aligned}\right.
$$

$i=1, \ldots, m, j=1, \ldots, l$. It is evident then that $T_{G}\left(x_{1}, \ldots, x_{l}\right)=\prod_{i=1}^{m}\left(a_{i 1} x_{1}+\ldots+a_{i l} x_{l}\right)$. (Note that vertex and edge numberings, hence the order of rows and columns in $A_{G}$ do not influence the permanent index.)

Lemma 5 Let $G$ be a simple graph with a given orientation, and let $A_{G}=\left(a_{i j}\right)$ be a matrix associated with it. Then $\operatorname{tmind}(G)=\operatorname{pind}\left(A_{G}\right)$.

Proof. Assume that $G$ is of size $m$ and order $n, l=m+n$, and has some fixed orientation. It is enough to note that the coefficient of each monomial of the form $x_{j_{1}}^{k_{1}} \ldots x_{j_{l}}^{k_{l}}$ in the expansion of $T_{G}\left(x_{1}, \ldots, x_{l}\right)=\prod_{i=1}^{m}\left(a_{i 1} x_{1}+\ldots+a_{i l} x_{l}\right)$ is equal to $\frac{\text { per } A_{G}(K)}{k_{1} ! \ldots k_{l} !}$, where $K=\left(k_{1}, \ldots, k_{l}\right)$.

To make more clear some of the introduced definitions note an example in picture 1 below. For such a graph $G$ with the given orientation, the associated polynomial $T_{G}$ is of the form $T_{G}\left(x_{1}, \ldots, x_{8}\right)=\left(x_{2}+x_{4}+x_{6}-x_{5}\right)\left(x_{3}+x_{7}-x_{1}-x_{4}-x_{6}\right)\left(x_{4}+x_{8}-x_{2}-\right.$ $\left.x_{7}\right)\left(x_{1}+x_{2}+x_{6}-x_{3}-x_{8}\right)$. Moreover, it is easy to check that $\operatorname{per}\left[A_{1}, A_{3}, A_{5}, A_{7}\right]=2 \neq 0$ 


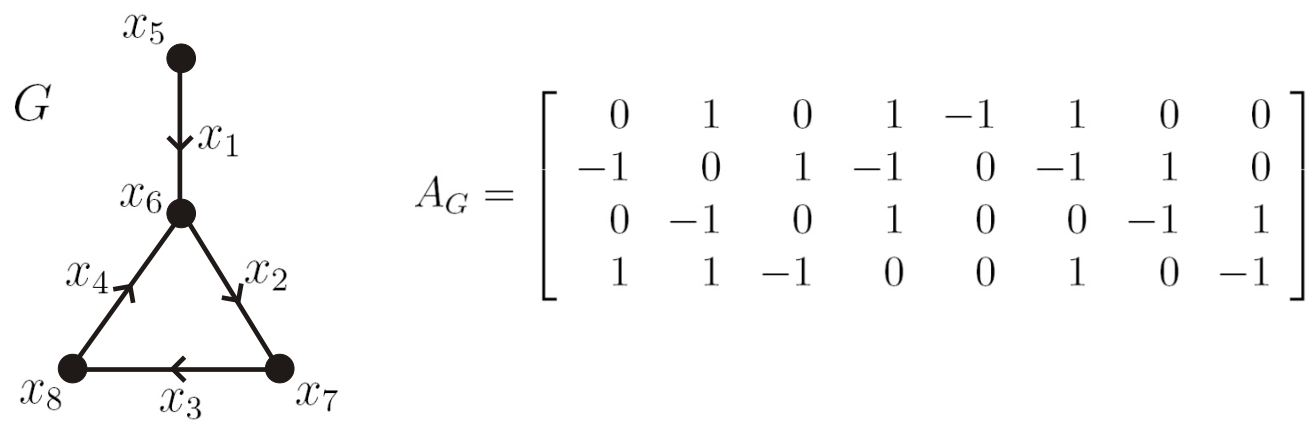

Figure 1: Example: An orientation of $G$ and an associated matrix.

for $A=A_{G}$, hence the coefficient of the monomial $x_{1} x_{3} x_{5} x_{7}$ in the expansion of $T_{G}$ equals 2 , $\operatorname{pind}(A)=1=\operatorname{tmind}(G)$ and $G$ is 2-totally weight choosable.

We shall conclude this section with one more, slightly technical, but very useful lemma, which, as well as lemma 5, corresponds with a similar one from the paper [4] of Bartnicki, Grytczuk and Niwczyk.

Lemma 6 Let $A$ be a matrix of size $m \times l$, and let $L$ be a square matrix of size $m$ such that each column of $L$ is a linear combination of columns of $A$. Let $n_{j}$ be the number of those columns of $L$ in which the $j$-th column of $A$ appears with non-zero coefficient. If $\operatorname{per} L \neq 0$ and $n_{j} \leq r$ for $j=1, \ldots, l$, then $\operatorname{pind}(A) \leq r$.

Proof. Let $L_{k}$ be the $k$-th column of $L$. By our assumption, $L_{k}=\sum_{j=1}^{l} b_{k j} A_{j}$. Since the permanent of a matrix is a multilinear function with respect to columns, we may expand $\operatorname{per} L$ as a sum of terms of the form $c \times\left(\operatorname{per}\left[A_{j_{1}}, \ldots, A_{j_{m}}\right]\right)$, where $c=\prod_{k=1}^{m} b_{k j_{k}}$. Since $\operatorname{per} L$ is non-zero, at least one of these terms must be non-zero. The statement therefore holds by our assumption $n_{j} \leq r$.

\section{Application of the method}

By observation 4, we may now use a result from [4] to easily obtain the subsequent corollary 8.

Theorem 7 ([4]) If $G \neq K_{2}$ is a clique, complete bipartite graph or a tree, then $\operatorname{mind}(G) \leq 2$.

Corollary 8 If $G$ is a clique, complete bipartite graph or a tree, then $\operatorname{tmind}(G) \leq 2$.

By corollary 3 we therefore obtain the following one.

Corollary 9 All cliques, complete bipartite graphs and trees are 3-totally weight choosable. 
An easy observation provides us with an improvement of this result for trees.

Lemma 10 Let $u$ be a vertex of a graph $G=(V, E)$ and assume $v \notin V$. Denote $G^{\prime}=$ $G+u v$. If $\operatorname{tmind}(G)=1$, then $\operatorname{tmind}\left(G^{\prime}\right)=1$.

Proof. Let $G$ be an oriented graph of size $m$ and order $n$, and let $A=A_{G}$ be a matrix associated with it. Then by lemma $5, \operatorname{per}\left[A_{j_{1}}, \ldots, A_{j_{m}}\right] \neq 0$ for some set of $m$ columns of $A$. Orient a new edge $e:(u, v)$, and let $A^{\prime}=A_{G^{\prime}}$ be a matrix associated with the obtained $G^{\prime}$. Then it is of the form

$$
A^{\prime}=\left[\begin{array}{c|c|c}
A & C_{e} & \vdots \\
& & 0 \\
\hline R_{e} & 0 & 1
\end{array}\right],
$$

where $R_{e}$ and $C_{e}$ are a row and a column corresponding with the newly added edge $e$, and the last column of $A^{\prime}$, which we denote $A_{l^{\prime}}^{\prime}$, corresponds with a new vertex $v$ (though according to the introduced notations, the column corresponding with $e$ should appear somewhere earlier in $A_{G^{\prime}}$, this does not change our reasoning, since an exchange of positions of columns does not influence the permanent of a matrix). Then it is easy to see that $\operatorname{per}\left[A_{j_{1}}^{\prime}, \ldots, A_{j_{m}}^{\prime}, A_{l^{\prime}}^{\prime}\right]=\operatorname{per}\left[A_{j_{1}}, \ldots, A_{j_{m}}\right] \neq 0$, hence again by lemma $5, \operatorname{tmind}\left(G^{\prime}\right)=1$.

Corollary 11 For each tree $T, \operatorname{tmind}(T)=1$.

Proof. This fact is obvious for a single isolated edge, hence, by lemma 10, the result follows by induction.

By the above corollary, each tree is 2-totally weight choosable. Though this fact is not difficult to show without Combinatorial Nullstellensatz, our algebraic approach surely helped to prove the subsequent results.

Theorem 12 For each $n, \operatorname{tmind}\left(C_{n}\right)=1$.

Proof. Given a cycle $G=C_{n}$ orient it clockwise, as in picture 2. Then its associated matrix is of the form

$$
A_{G}=\left[\begin{array}{cccccc|cccccc}
0 & 1 & 0 & & 0 & -1 & -1 & 1 & 0 & & 0 & 0 \\
-1 & 0 & 1 & \ldots & 0 & 0 & 0 & -1 & 1 & \ldots & 0 & 0 \\
0 & -1 & 0 & & 0 & 0 & 0 & 0 & -1 & & 0 & 0 \\
& \vdots & & \ddots & \vdots & & & \vdots & & \ddots & \vdots & \\
0 & 0 & 0 & \ldots & 0 & 1 & 0 & 0 & 0 & \ldots & -1 & 1 \\
1 & 0 & 0 & & -1 & 0 & 1 & 0 & 0 & & 0 & -1
\end{array}\right]
$$

where the first $n$ columns correspond with the subsequent edges of the cycle, while the remaining ones correspond with its $n$ vertices. 


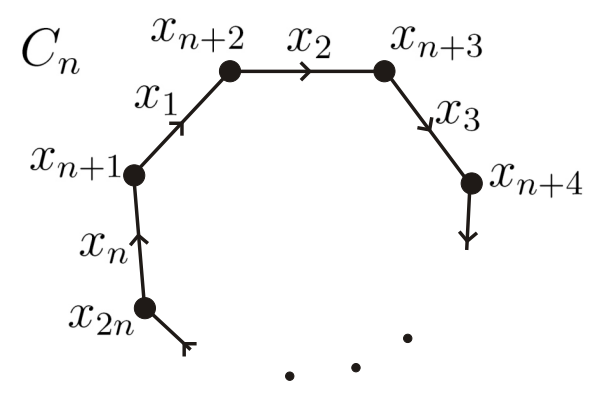

Figure 2: An orientation of a cycle.

Assume first that $n$ is even and take a square submatrix of $A_{G}$ of size $n$ consisting of the $n$ last rows of $A_{G}$. Then an application of the Laplace expansion for permanents with respect to the first column of this matrix yields what follows.

$$
\begin{gathered}
\operatorname{per}\left[\begin{array}{cccccc}
-1 & 1 & 0 & & 0 & 0 \\
0 & -1 & 1 & \cdots & 0 & 0 \\
0 & 0 & -1 & & 0 & 0 \\
& \vdots & & \ddots & \vdots & \\
0 & 0 & 0 & \cdots & -1 & 1 \\
1 & 0 & 0 & & 0 & -1
\end{array}\right]=- \text { per }\left[\begin{array}{ccccc}
-1 & 1 & & 0 & 0 \\
0 & -1 & \cdots & 0 & 0 \\
& \vdots & \ddots & \vdots & \\
0 & 0 & \cdots & -1 & 1 \\
0 & 0 & & 0 & -1
\end{array}\right] \\
\quad+\operatorname{per}\left[\begin{array}{ccccc}
1 & 0 & & 0 & 0 \\
-1 & 1 & \cdots & 0 & 0 \\
& \vdots & \ddots & \vdots & \\
0 & 0 & \cdots & 1 & 0 \\
0 & 0 & & -1 & 1
\end{array}\right]=-(-1)^{n-1}+1^{n}=1+1=2 \neq 0 .
\end{gathered}
$$

Now suppose that $n$ is odd and take a square submatrix of $A_{G}$ consisting of the $n$-th row and the $(n-1)$ last rows of $A_{G}$. Again an application of the Laplace expansion with 
respect to the first column of this matrix results in the following.

$$
\begin{gathered}
\operatorname{per}\left[\begin{array}{cccccc}
-1 & 1 & 0 & & 0 & 0 \\
0 & -1 & 1 & \cdots & 0 & 0 \\
0 & 0 & -1 & & 0 & 0 \\
& \vdots & & \ddots & \vdots & \\
1 & 0 & 0 & \cdots & -1 & 1 \\
0 & 0 & 0 & & 0 & -1
\end{array}\right]=-\operatorname{per}\left[\begin{array}{ccccc}
-1 & 1 & & 0 & 0 \\
0 & -1 & \cdots & 0 & 0 \\
& \vdots & \ddots & \vdots & \\
0 & 0 & \cdots & -1 & 1 \\
0 & 0 & & 0 & -1
\end{array}\right] \\
+\operatorname{cocer}\left[\begin{array}{ccccc}
1 & 0 & & 0 & 0 \\
-1 & 1 & \cdots & 0 & 0 \\
& \vdots & \ddots & \vdots & \\
0 & 0 & \cdots & 1 & 0 \\
0 & 0 & & 0 & -1
\end{array}\right]=-(-1)^{n-1}+(-1)=-1-1=-2 .
\end{gathered}
$$

In both cases, by lemma $5, \operatorname{tmind}\left(C_{n}\right)=1$.

By lemma 10 and theorem 12 we can now easily prove the following corollary using induction.

Corollary 13 Let $G$ be a unicyclic graph. Then $\operatorname{tmind}(G)=1$.

The last result of this section exemplifies an application of lemma 6 .

Theorem 14 For each wheel $W_{n}$ with $n+1$ vertices, $\operatorname{tmind}\left(W_{n}\right)=1$.

Proof. Given a wheel $G=W_{n}$ orient it as in picture 3. Then its associated matrix

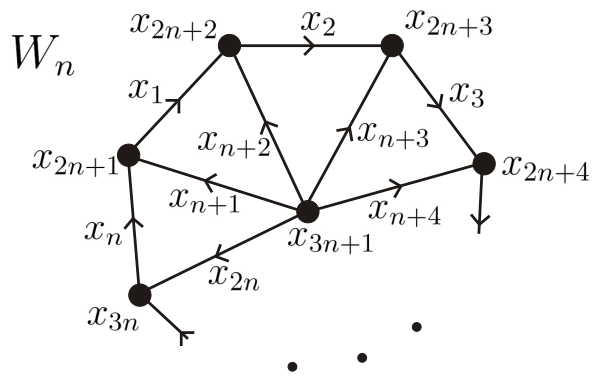

Figure 3: An orientation of a wheel. 
$A=A_{G}$ is of the form

$$
A=\left[\begin{array}{ccccc|ccccc|ccccc|c}
0 & 1 & 0 & & -1 & -1 & 1 & 0 & & 0 & -1 & 1 & 0 & & 0 & 0 \\
-1 & 0 & 1 & \ldots & 0 & 0 & -1 & 1 & \ldots & 0 & 0 & -1 & 1 & \ldots & 0 & 0 \\
0 & -1 & 0 & & 0 & 0 & 0 & -1 & & 0 & 0 & 0 & -1 & & 0 & 0 \\
& \vdots & & \ddots & \vdots & & \vdots & & \ddots & \vdots & & \vdots & & \ddots & \vdots & \vdots \\
1 & 0 & 0 & \ldots & 0 & 1 & 0 & 0 & \ldots & -1 & 1 & 0 & 0 & \ldots & -1 & 0 \\
\hline 1 & 0 & 0 & & 1 & 0 & -1 & -1 & & -1 & 1 & 0 & 0 & & 0 & -1 \\
1 & 1 & 0 & \ldots & 0 & -1 & 0 & -1 & \ldots & -1 & 0 & 1 & 0 & \ldots & 0 & -1 \\
0 & 1 & 1 & & 0 & -1 & -1 & 0 & & -1 & 0 & 0 & 1 & & 0 & -1 \\
& \vdots & & \ddots & \vdots & & \vdots & & \ddots & \vdots & & \vdots & & \ddots & \vdots & \vdots \\
0 & 0 & 0 & \ldots & 1 & -1 & -1 & -1 & \ldots & 0 & 0 & 0 & 0 & \ldots & 1 & -1
\end{array}\right],
$$

where the first $n$ columns correspond with the subsequent edges of the outer cycle, the next $n$, with the spokes of the wheel, and the remaining $n+1$ ones are associated with the vertices (where the last one corresponds with the center vertex). Similarly, the first $n$ rows is associated with the edges of the cycle, and the remaining ones, with the spokes.

Let us define the following matrix $L=\left[A_{2}, A_{3}, \ldots, A_{n-1}, A_{2 n}, A_{3 n}, A_{2 n+1}-A_{n+1}, A_{2 n+2}-\right.$ $\left.A_{n+2}, \ldots, A_{3 n-1}-A_{2 n-1},-A_{3 n+1}\right]$. Then we have

$$
\operatorname{per} L=\operatorname{per}\left[\begin{array}{cccccc|cc|cccccccc}
1 & 0 & 0 & 0 & & 0 & 0 & 0 & 0 & 0 & 0 & 0 & & 0 & 0 & 0 \\
0 & 1 & 0 & 0 & & 0 & 0 & 0 & 0 & 0 & 0 & 0 & & 0 & 0 & 0 \\
-1 & 0 & 1 & 0 & \cdots & 0 & 0 & 0 & 0 & 0 & 0 & 0 & \cdots & 0 & 0 & 0 \\
0 & -1 & 0 & 1 & & 0 & 0 & 0 & 0 & 0 & 0 & 0 & & 0 & 0 & 0 \\
& & \vdots & & \ddots & \vdots & \vdots & \vdots & & & \vdots & & \ddots & & \vdots & \\
0 & 0 & 0 & 0 & \cdots & 1 & 0 & 0 & 0 & 0 & 0 & 0 & \cdots & 0 & 0 & 0 \\
\hline 0 & 0 & 0 & 0 & \cdots & 0 & 1 & 1 & 0 & 0 & 0 & 0 & \cdots & 0 & 0 & 0 \\
0 & 0 & 0 & 0 & \cdots & -1 & -1 & -1 & 0 & 0 & 0 & 0 & \cdots & 0 & 0 & 0 \\
\hline 0 & 0 & 0 & 0 & & 0 & -1 & 0 & 1 & 1 & 1 & 1 & & 1 & 1 & 1 \\
1 & 0 & 0 & 0 & & 0 & -1 & 0 & 1 & 1 & 1 & 1 & & 1 & 1 & 1 \\
1 & 1 & 0 & 0 & \cdots & 0 & -1 & 0 & 1 & 1 & 1 & 1 & \cdots & 1 & 1 & 1 \\
0 & 1 & 1 & 0 & & 0 & -1 & 0 & 1 & 1 & 1 & 1 & & 1 & 1 & 1 \\
& & \vdots & & \ddots & \vdots & \vdots & \vdots & & & \vdots & & \ddots & & \vdots & \\
0 & 0 & 0 & 0 & & 0 & -1 & 0 & 1 & 1 & 1 & 1 & & 1 & 1 & 1 \\
0 & 0 & 0 & 0 & \cdots & 1 & -1 & 0 & 1 & 1 & 1 & 1 & \cdots & 1 & 1 & 1 \\
0 & 0 & 0 & 0 & & 1 & 0 & 1 & 1 & 1 & 1 & 1 & & 1 & 1 & 1
\end{array}\right]
$$

Note that the columns of $L$ are linear combinations of columns of $A$ and neither of the columns of $A$ appears more than once in these linear combinations. Therefore, by lemma $6, \operatorname{tmind}(G)=1$. 


\section{Complete graphs}

In the previous section we proved our conjecture for trees, wheels and unicyclic graphs (and a slightly weaker result for complete and complete bipartite graphs), what, in the case of trees, was an improvement of corollary 9. In this section we shall improve this corollary and prove conjecture 1 in the case of complete graphs. For this aim we shall not use an algebraic method. Let $V=\left\{v_{1}, \ldots, v_{n}\right\}$ and $E=\left\{e_{1}, \ldots, e_{m}\right\}$ be the vertex set and the edge set of a graph $G$, and assume we are given a collection $L=\left\{L_{v_{1}}, \ldots, L_{v_{n}}, L_{e_{1}}, \ldots\right.$, $\left.L_{e_{m}}\right\}$ of lists of size $k$ assigned to the vertices and the edges of $G$. For each list $L_{a} \in L$, let $m_{L}(a)=\min L_{a}$ and $M_{L}(a)=\max L_{a}$. Now define the minimal potential total weight and the maximal potential total weight of a vertex with respect to $L$ as

$$
\begin{aligned}
& m(L)=\min \left\{\sum_{e \ni v} m_{L}(e)+m_{L}(v): v \in V\right\}, \\
& M(L)=\max \left\{\sum_{e \ni v} M_{L}(e)+M_{L}(v): v \in V\right\} .
\end{aligned}
$$

For technical reasons, we shall prove a slightly stronger statement.

Theorem 15 Let $L$ be a collection of lists of size 2 assigned to the vertices and the edges of a complete graph $G=K_{n}$. There are (at least) two neighbour distinguishing total weightings of $G$ with weights from the specified lists from $L$, one without a total weight $m(L)$, and the other without a total weight $M(L)$ among all the vertices of $G$.

Proof. It is easy to observe that the theorem holds for $n \leq 2$. Assume then that $G=K_{n}=$ $(V, E)$ has at least three vertices. We prove the statement by induction with respect to $n$. Let $L$ comply with the requirements of the theorem for $G$. Now choose any vertex $v \in V$ that realizes the minimal potential total weight $m(L)$ (i.e. $\sum_{e \ni v} m_{L}(e)+m_{L}(v)=m(L)$ ). Let $G^{\prime}=G-v$ and let us define a new collection $L^{\prime}$ of lists of size 2 for $G^{\prime}=\left(V^{\prime}, E^{\prime}\right)$ in the following way. The lists of the edges do not change (except for the fact that there are $(n-1)$ less of such lists). For each vertex $u \in V^{\prime}$ on the other hand, let

$$
L_{u}^{\prime}=\left\{m_{L}(u)+m_{L}(u v), M_{L}(u)+m_{L}(u v)\right\} .
$$

Note that $m\left(L^{\prime}\right) \geq m(L)$. By our induction assumption, we may now find a neighbour distinguishing total weighting $w^{\prime}$ of $G^{\prime}$ with weights from the specified lists from $L^{\prime}$, such that $t_{w^{\prime}}(u)>m\left(L^{\prime}\right)$ for each $u \in V^{\prime}$. Let us next define a total weighting $w$ of $G$ in the following way. For each $e \in E^{\prime}, w(e)=w^{\prime}(e)$. For each vertex $u \in V^{\prime}, w(u)=$ $w^{\prime}(u)-m_{L}(u v)$. For each edge $u v \in E \backslash E^{\prime}, w(u v)=m_{L}(u v)$, and $w(v)=m_{L}(v)$. Then for each $u \in V^{\prime}, t_{w}(u)=t_{w^{\prime}}(u)>m(L)$, hence the vertices from $V^{\prime}$ remain distinguished from their neighbours in $V^{\prime}$. On the other hand, $t_{w}(v)=m(L)$ and all the weights of $w$ belong to the specified lists from $L$, hence we obtain a desired total weighting $w$ of $G$ without a total weight $M(L)$ (because each vertex $u \in V$ is adjacent with at least one edge $e$ weighted with a minimal number from the list $L_{e}$ ). 
It is now an exercise to repeat an analogous reasoning to prove that if $v$ realizes the maximal potential total weight $M(L)$, then we can construct an expected total weighting $w$ of $G$ without a total weight $m(L)$.

Acknowledgements. The authors inform that their research were partially supported by the Polish Ministry of Science and Higher Education, grant no. N201 1247/33.

\section{References}

[1] L. Addario-Berry, K. Dalal, B.A. Reed, Degree Constrained Subgraphs, Discrete Appl. Math. 156 (7) (2008) 1168-1174.

[2] N. Alon, Combinatorial Nullstellensatz, Combin. Probab. Comput. 8 (1999) 7-29.

[3] M. Bača, S. Jendrol̆, M. Miller, J. Ryan, On Irregular Total Labelings, Discrete Math. 307 (2007) 1378-1388.

[4] T. Bartnicki, J. Grytczuk, S. Niwczyk, Weight Choosability of Graphs, J. Graph Theory. 60 (3) (2009) 242-256.

[5] G.J. Chang, T. Wong, X. Zhu, Total weight choosability of trees, manuscript.

[6] G. Chartrand, M.S. Jacobson, J. Lehel, O.R. Oellermann, S. Ruiz, F. Saba, Irregular Networks, Proc. of the 250th Anniversary Conf. on Graph Theory, Congr. Numer. 64 (1988) 197-210.

[7] A. Frieze, R.J. Gould, M. Karoński, F. Pfender, On Graph Irregularity Strength, J. Graph Theory 41 (2) (2002) 120-137.

[8] M. Kalkowski, A note on 1,2-Conjecture, to appear in Elect. Journ. of Comb.

[9] M. Karoński, T. Łuczak, A. Thomason, Edge weights and vertex colours, J. Combin. Theory, Ser. B 91 (2004) 151-157.

[10] T. Nierhoff, A tight bound on the irregularity strength of graphs, SIAM J. Discrete Math. 13 (3) (2000) 313-323.

[11] J. Przybyło, A note on a neighbour-distinguishing regular graphs total-weighting, Electron. J. Combin. 15 (1) (2008) $\sharp$ N35.

[12] J. Przybyło, Irregularity strength of regular graphs, Electron. J. Combin. 15 (1) (2008) $\sharp R 82$.

[13] J. Przybyło, Linear bound on the irregularity strength and the total vertex irregularity strength of graphs, SIAM J. Discrete Math. 23 (1) (2009) 511-516.

[14] J. Przybyło, M. Woźniak On a 1,2 Conjecture, Discrete Math. Theor. Comput. Sci. 12:1 (2010) 101-108.

[15] T. Wong, D. Yang, X. Zhu, Total weighting of graphs by max-min method, to appear in a volume dedicated to Lovász's 60th birthday, Bolyai Society Mathematical Studies (2009).

[16] T. Wong and X. Zhu, Total weight choosability of graphs, J. Graph Theory 66 (2011) $198-212$. 